\title{
Upslope Flows in Atmosphere and Water Tank, Part II: Fluid-Dynamical Smoothness as a Possible Cause for Velocity Similarity Violation
}

\author{
C. Reuten ${ }^{*}$, S. E. Allen, and D. G. Steyn \\ Atmospheric Science Program, Department of Earth and Ocean Sciences, The University of British Columbia, \\ Vancouver, Canada
}

\begin{abstract}
Water-tank models of meso-scale atmospheric processes often show good qualitative agreement of bulk quantities and flow characteristics and good quantitative agreement of turbulence quantities with field observations. However, it was demonstrated in the first part of this two-part communication that the similarity of velocities of thermally driven upslope flows in atmosphere and water tank is violated.

It is shown in this part that the velocities of thermally driven upslope flows in the atmosphere and in a water-tank model have statistically different dependences on proposed governing parameters. Of four substantially different hypotheses of upslope velocities, three agree with field observations because of large uncertainties and sparse data, but all hypotheses disagree with tank observations. One hypothesis that includes the influence of the total slope height agrees with field and tank observations when assuming fluid-dynamically rough atmospheric flows and fluid-dynamically smooth tank flows. The non-dimensional upslope flow velocities corresponding to rough and smooth flows depend differently on the governing parameters. Therefore, non-dimensional upslope flow velocities are different for atmosphere and water tank. Furthermore, as this hypothesis includes a dependence of the upslope flow velocity on the total height of the slope it implies that upslope flow systems are non-local phenomena.

Because fluid-dynamical roughness is technically difficult to achieve in water-tank models, velocity similarity violations can also be expected in water-tank models of other thermally driven meso-scale flows and our technique of explicitly including roughness length dependence may have wider applications.
\end{abstract}

Keywords: Bayesian analysis, physical scale model, scaling, similarity, upslope flows, water tank.

\section{INTRODUCTION}

Water-tank models have been used for several decades to study atmospheric phenomena. Some of the earlier experiments focused primarily on turbulence in the convective boundary layer (CBL) in salt- or heat-stratified water over a heated flat horizontal plate [1-6] and the role of turbulence in dispersion of pollutants [7-20]. Many of these studies investigated the CBL under externally imposed advection. Examples of thermally driven flows that have been studied with water-tank models are sea breezes [21], urban heat islands [22, 23] and their interactions [24], and upslope flows [25-27], which are the focus here. Simply speaking, upslope flows arise over heated sloping terrain in a stratified background fluid; fluid near the slope surface will be warmer than fluid at the same elevation further from the slope. Buoyancy and pressure-gradient forces together cause an upslope flow approximately parallel to the slope. This paper is the second part of a two-part communication. From here on we will refer to part I and part II for simplicity.

Previous tank studies of upslope flows showed similarity between atmosphere and water tank for turbulence parameters [28], flow characteristics [25, 29], and the order

*Address correspondence to this author at the RWDI AIR Inc., Suite 1000, $7368^{\text {th }}$ Ave. SW, Calgary, AB T2P 1H4, Canada; Tel: 1-403-232-6771; Fax.: 1-403-232-6762; Email: creuten@gmail.com of magnitude of bulk quantities like CBL height and upslope flow velocity [26, 27]. In part $I$, it was shown that quantitative similarity can be achieved for CBL depth within approximately the uncertainty of field and tank observations $(20 \%)$ but that upslope flow velocities are significantly different. Here in part II, the cause for the similarity violation of upslope flow velocity will be studied. A summary of the scaling and a description of the field and tank experiments are provided in section 2. In section 3, four alternative upslope flow velocity hypotheses are developed. In section 4 these are compared with field and tank observations using Bayesian analysis. A detailed discussion of the results is presented in section 5, and conclusions are drawn in section 6. The derivation of the equations used for the Bayesian analysis is provided in the appendix. Quantities in the atmosphere will be denoted with subscript ' $a$ ' to distinguish them from those in the water tank with subscript 'w'; no subscripts will be used if the quantity or equation applies to both atmosphere and water tank.

\section{METHODS}

\section{a. Field Observations}

Details of the field site and instrumentation at Minnekhada Park in the Lower Fraser Valley, British Columbia, Canada, are presented in [30]. The slope is fairly homogeneous over a width of roughly $3 \mathrm{~km}$; the slope angle is approximately constant at $19^{\circ}$ and ridge height is $760 \mathrm{~m}$. 
Figure 2 in [30] suggests that a plateau better represents the terrain on the other side of the ridge than a slope. The plain adjacent to the slope is approximately flat. Observations used in this paper were taken under mostly cloudless skies in the morning hours of 25-26 July 2001, before the onset of sea-breeze and up-valley flows. Vertical profiles of moisture and temperature were measured with a tethersonde $3.5 \mathrm{~km}$ from the bottom of the slope. Early morning profiles showed roughly constant lapse rates up to about $1000 \mathrm{~m}$, except very near the surface. Range-height indicator (RHI) scans with a lidar provided information on the backscatter boundary layer (BBL) depth over plain and slope, which reached values of about $800 \mathrm{~m}$. Over the plain close to the slope a Doppler sodar was employed to measure vertical profiles of wind velocity averaged over approximately 20 minutes. 'Upslope flow velocities' were determined from each vertical profile as the maximum value of the horizontal component of the slope wind, typically 3-6 $\mathrm{m} \mathrm{s}^{-1}$.

\section{b. Water Tank}

The water tank (Fig. 1) has glass walls and a stainless steel bottom encased in a stainless steel frame. Because field observations showed fairly constant lapse rate all water-tank experiments were started with linear salt stratification. Convection is triggered in the tank by heating the bottom steel plate from below with strip heaters, which can be controlled individually or in groups of five to six heaters. Because heating could only be varied manually within 40$100 \%$ of the maximum power supply, all but one experiment were carried out with steady heat flux (Table 1). In experiment WT4, heat flux was manually controlled to approximate the roughly sinusoidal surface heat flux development found in the atmosphere. The experiments in Table 1 cover a wide range of parameter values and differ substantially in plain/plateau length and spatial and temporal variations of heat flux at the slope surface.

Two-dimensional velocity fields were measured from the motion of neutrally buoyant particles using the PIV ('particle image velocimetry') toolbox for Matlab ${ }^{\circledR}$. Maximum upslope flow velocities were computed in two steps. First, medians over 20-s intervals were calculated to remove turbulent variations. Medians successfully removed outliers and were faster to calculate than means with outlier filters. Second, the medians of the maxima in three adjacent vertical profiles of horizontal velocities centered over the slope $20 \mathrm{~cm}$ upslope from its base were calculated to further smooth the data. The use of the median rather than the mean slightly reduced the influence of some extreme values. The results were very robust when averaging time was doubled and when five rather than three adjacent profiles were used. Experiments lasted roughly $1000 \mathrm{~s}$. Maximum horizontal velocities ranged from $0.5-2.0 \mathrm{~cm} \mathrm{~s}^{-1}$. More technical details can be found in [29].

\section{c. Scaling}

This section provides a summary of the scaling that was performed in part I of this paper. Mathematical idealizations were formulated for the atmosphere and the water tank and ten potentially relevant dimensional parameters were

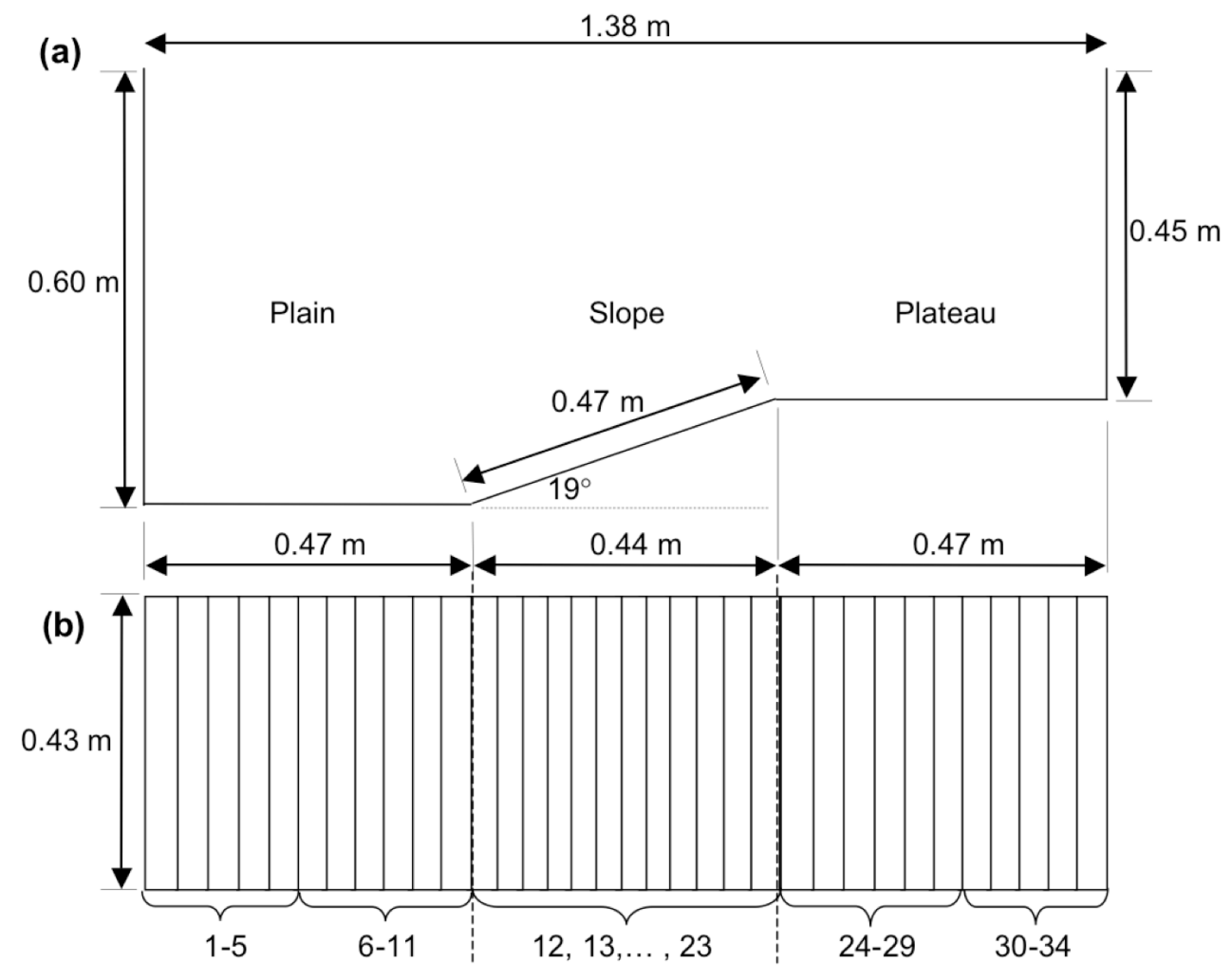

Fig. (1). Schematic of the water tank. Scale is approximately 1:10. (a) Side view. (b) Plan view with strip heaters underneath the tank bottom numbered from 1 to 34 . Heaters can be controlled individually underneath the slope and in two groups of five to six heaters each underneath plain and plateau as indicated. 
Table 1. Overview of Water-Tank Experiments Used for Upslope Flow Velocities Analyses

\begin{tabular}{|c|c|c|c|c|c|c|c|c|}
\hline \multirow{2}{*}{ Name } & \multirow{2}{*}{$N_{w}\left(\mathbf{s}^{-1}\right)$} & \multirow{2}{*}{$\bar{Q}_{H, w}\left(10^{-3} \mathrm{~K} \mathrm{~m} \mathrm{~s}^{-1}\right)$} & \multirow{2}{*}{$\Pi_{3, w}$} & \multirow{2}{*}{$L_{b, w}(\mathbf{m})$} & \multirow{2}{*}{$L_{t, w}(\mathbf{m})$} & \multicolumn{3}{|c|}{$Q_{H, w}$ Details $\left(10^{-3} \mathrm{~K} \mathrm{~m} \mathrm{~s}^{-1}\right)$} \\
\hline & & & & & & Plain & Slope & Plateau \\
\hline WT1 & 0.567 & 1.85 & 0.00117 & 0.470 & 0.470 & 1.85 & 1.85 & 1.85 \\
\hline WT2 & 0.379 & 1.85 & 0.00406 & 0.470 & 0.470 & 1.85 & 1.85 & 1.85 \\
\hline SP & 0.379 & 1.85 & 0.00406 & 0.225 & 0.470 & 1.85 & 1.85 & 1.85 \\
\hline TR1 & 0.379 & 2.68 & 0.00588 & 0.470 & 0 & 1.48 & $1.67-3.70$ & - \\
\hline TR2 & 0.342 & 3.15 & 0.00903 & 0.470 & 0 & $1.48 / 2.04$ & $2.59-3.70$ & - \\
\hline WT3 & 0.374 & 2.96 & 0.00649 & 0.470 & 0.470 & 1.85 & 2.96 & 1.85 \\
\hline WT4 & 0.423 & $1.48-3.52$ & $0.00219-0.00521$ & 0.470 & 0.470 & $1.48-3.52$ & $1.48-3.52$ & $1.48-3.52$ \\
\hline
\end{tabular}

Experiments are named according to their geometry as: WT ('Whole Tank'), SP ('Short Plain', additional end wall over the plain $L_{b, w}=0.225 \mathrm{~m}$ from the slope), and TR ('Triangular Ridge', additional end wall at the ridge top, $L_{t, w}=0$ ); numbers distinguish experiments with equal geometry. $\Pi_{3, w}$ is computed for $\bar{Q}_{H, w}$, the surface heat flux spatially averaged over the slope only. The last three columns show details of the heat flux supplied to the tank. In TR1 the heat flux at the slope surface increased with height along the slope in twelve equal increments from 1.67 to $3.70 \times 10^{-3} \mathrm{~K} \mathrm{~m} \mathrm{~s}^{-1}$. In TR2, the slope surface heat flux increased from 2.39 to $3.70 \times 10^{-3} \mathrm{~K} \mathrm{~m} \mathrm{~s}^{-1}$, and the surface heat flux in left and right half of the plain was 1.48 and $2.04 \times 10^{-3} \mathrm{~K} \mathrm{~m} \mathrm{~s}^{-1}$, respectively. In WT4, heat flux was stepped up manually to roughly follow sinusoidal time development.

identified for the atmosphere and an additional two for the water tank: Ridge height $H$, sensible surface heat flux $Q_{H}$, buoyancy frequency $N$, horizontal length of slope $L$, total supplied energy density $E$, buoyancy parameter $g \beta$ ( $g$ is the gravitational acceleration and $\beta$ the coefficient of thermal expansion), kinematic viscosity $v$, thermal diffusivity $\kappa$, length of the plain $L_{b}$, length of the plateau $L_{t}$, and roughness height $Z_{r}$; in addition for the water tank its width $W_{w}$ and the water depth over the plain $D_{w}$. We speculated in part I that the difference in Reynolds number, at the roughness height, may play an important role for the upslope flow velocity violation. In fluid-dynamically rough flow, the roughness elements lead to momentum roughness lengths much greater than thermal roughness lengths [31]. If the Reynolds number, at the scale of the roughness, is too small, molecular properties dominate and the appropriate momentum length and thermal length will be more similar. Thus, in part II, we change from using $Z_{r}$ as a parameter, to introducing two separate parameters, the momentum $z_{m}$ and thermal $z_{T}$ roughness lengths. The Buckingham Pi Theorem [32] implies that the three involved fundamental units $(\mathrm{K}, \mathrm{m}, \mathrm{s})$ require three independent key parameters, which were chosen to be $H, Q_{H}$, and $N$, to non-dimensionalize the remaining parameters and form nine atmospheric and eleven water-tank Pi Groups (Table 2).

Convective boundary layer depth $h$ is nondimensionalized by dividing by $H, h^{*}=h / H$, and maximum upslope flow velocity $U$ is non-dimensionalized by dividing by $H N, U^{*}=U /(H N)$. In the first part it was proposed that $h^{*}$ and $U^{*}$ only depend on the aspect ratio $\Pi_{1}=L / H$, nondimensional (ND) energy density $\Pi_{2}=E N / Q_{H}$, and ND buoyancy parameter $\Pi_{3}=g \beta Q_{H} /\left(H^{2} N^{3}\right)$. Analyses of atmospheric and tank observations showed similarity of $\mathrm{CBL}$ depth and a similarity violation of upslope flow velocity. The value of the aspect ratio $\Pi_{1}$ is the same for the idealized mathematical models of field site ('atmospheric idealization') and water-tank ('water-tank idealization') and can therefore not be responsible for the similarity violation. In this paper it will be investigated if the exponents $m_{1}$ and $m_{2}$ in the assumed monomial relationship $U^{*}=c \Pi_{2}^{m_{1}} \Pi_{3}^{m_{2}}$ are the same for atmospheric and tank observations. If they are the same then, because $U^{*}$ differs, the coefficient $c$ would have to be different and therefore depend not only on the aspect ratio $\Pi_{1}$ but also on other Pi groups, which are different for atmosphere and water tank. Before the functional dependence of $U^{*}$ on $\Pi_{2}$ and $\Pi_{3}$ is further investigated, alternative hypotheses of upslope flow velocities are developed from the literature.

\section{UPSLOPE FLOW VELOCITY HYPOTHESES}

Four hypotheses of upslope flow velocities in atmosphere and water tank are proposed. Three are modifications of hypotheses extracted from the literature and for easy reference are named after the first author of the publications. To facilitate a comparison of the hypotheses, the notation is adapted to be the same across all hypotheses. The goal is to express the model predictions for ND upslope flow velocity in the form $U^{*}=c \Pi_{2}^{m_{1}} \Pi_{3}^{m_{2}}$. Further assumptions are necessary to bring the equations into this form. Most hypotheses include CBL depth $h$ as a known external parameter. We treat $h$ as an externally forced quantity and express it in terms of total supplied energy $E$. The assumption of an encroachment model [33] in part I led to similarity of CBL in atmosphere and water tank within expected uncertainties of $20 \%$. The more realistic entrainment model [34] would only lead to improvements if further research could establish the dependence of the entrainment coefficient on the Pi groups $\Pi_{2}$ and $\Pi_{3}$. Heidt 
Table 2. Summary of Pi Groups for Idealizations of the Atmosphere and the Water Tank. The Distinguishing Subscripts ' $a$ ' and ' $w$ ' were Dropped for Pi Groups that Apply to Both Idealizations

\begin{tabular}{|c|c|}
\hline Name & Symbol \\
\hline \multicolumn{2}{|c|}{ Core Pi Groups } \\
\hline Aspect ratio & $\Pi_{1}=\frac{L}{H}$ \\
\hline ND energy density or time & $\Pi_{2}=\frac{E N}{Q_{H}}$ \\
\hline ND buoyancy parameter / heat flux & $\Pi_{3}=\frac{g \beta Q_{H}}{H^{2} N^{3}}$ \\
\hline \multicolumn{2}{|c|}{ Molecular Pi Groups } \\
\hline ND viscosity & $\Pi_{4}=\frac{v}{H^{2} N}$ \\
\hline ND thermal diffusivity & $\Pi_{5}=\frac{\kappa}{H^{2} N}$ \\
\hline \multicolumn{2}{|c|}{ Longitudinal Boundary Conditions } \\
\hline ND half length of plain & $\Pi_{6}=\frac{L_{b}}{H}$ \\
\hline ND half length of plateau & $\Pi_{7}=\frac{L_{t}}{H}$ \\
\hline \multicolumn{2}{|c|}{ Water-Tank Specific Pi Groups } \\
\hline ND tank width & $\Pi_{8, w}=\frac{W_{w}}{H_{w}}$ \\
\hline ND water depth & $\Pi_{9, w}=\frac{D_{w}}{H_{w}}$ \\
\hline \multicolumn{2}{|c|}{ Roughness Length Pi Groups } \\
\hline ND momentum roughness length & $\Pi_{10}=\frac{z_{m}}{H}$ \\
\hline ND thermal roughness length & $\Pi_{11}=\frac{z_{T}}{H}$ \\
\hline
\end{tabular}

[4] suggested a constant entrainment coefficient, but this is questioned by more recent research [35], and the additional shear caused by the upslope flow circulation further complicates the issue. For an encroachment model,

$$
E_{a}=\frac{h_{a} \theta_{s, a}}{2} \text { and } E_{w}=\frac{h_{w} \alpha_{s, w}}{2 \alpha_{0, w} \beta_{w}}
$$

where $\theta_{s, a}$ is the potential temperature increase of the mean CBL potential temperature over the initial surface temperature and $\alpha_{s, w}$ is the specific volume increase of the mean CBL specific volume over the initial surface specific volume $\alpha_{0, w}$. Background stratifications in the encroachment model are $\gamma_{a}=\frac{\theta_{s, a}}{h_{a}}$ and $\gamma_{w}=\frac{\alpha_{s, w}}{\alpha_{0, w} \beta_{w} h_{w}}$.

In both atmosphere and tank, these are related to the buoyancy frequencies by:

$N=(g \beta \gamma)^{1 / 2}$.

Substituting $\gamma$ from (3) into (2), and the resulting equations for $\theta_{s, a}$ and $\alpha_{s, w}$ into (1), gives

$h=\left(\frac{2 g \beta E}{N^{2}}\right)^{1 / 2}$

for atmosphere and water tank.

\section{a. Hunt hypothesis}

Hunt et al. [27] allowed for different dynamics in the surface, mixed, and inversion layers. They treated each layer separately and constrained parameters by smoothly matching adjacent layers at their common boundaries. They derived simplified forms of the Navier-Stokes equations for the different layers and matched unknown parameters at the interfaces. For the mean upslope flow velocity they derived

$U_{M, a} \approx \Gamma_{a}\left(\sin \varphi \cdot g \beta_{a} h_{a} Q_{H, a}\right)^{1 / 3}$.

Hunt et al. [27] assumed slope angles $\varphi \leq 20^{\circ}$, approximated $\sin \varphi \approx \varphi$, and estimated

$\Gamma_{a} \sim \frac{1}{k} \ln \left(\frac{-L_{*, a}}{z_{m, a}}\right)\left[\frac{h_{s, a}}{h_{a}} \frac{1}{k} \ln \left(\frac{-L_{*, a}}{z_{T, a}}\right)\right]^{1 / 3}$,

where $\sim$ means 'is of same order of magnitude', $L_{*, a}$ is the Monin-Obukhov length, $h_{s, a}$ the surface layer depth, and $k \approx 0.4$ the von Kármán constant. Underlying (5) are the assumptions that

I. CBL and upslope flow layer are identical;

II. a deep unidirectional flow extends from the plain over the slope to the plateau without a return flow;

III. the environmental stratification above the upslope flow layer is undisturbed;

IV. the upslope flow velocity is independent of height within the mixed layer and is only slightly smaller than the maximum upslope flow velocity, which occurs in the surface layer; and

V. Monin-Obukhov theory is valid over sloping terrain and Monin-Obukhov length and surface layer depth are independent of upslope flow characteristics.

The functional dependence of the coefficient $\Gamma_{a}$ on its arguments in (6) and the values of the arguments are known only within order of magnitude. The dependence of $\Gamma_{a}$ on momentum and thermal roughness lengths implies that ND maximum upslope flow velocity depends on $\Pi_{10}$ and $\Pi_{11}$ (Table 2). This will be discussed further in section 5 . 
Initially, we will follow the suggestions in [27] that (5) also applies to upslope flows in water tanks and that $\Gamma \sim 10$ is roughly constant, therefore the dependence of $U^{*}$ on $\Pi_{10}$ and $\Pi_{11}$ is negligible. Substituting (4) into (5) and using the definitions of the Pi groups in Table 2 gives

$U_{H u n t} *=\frac{U_{H u n t}}{H N}=c_{H u n t} \cdot \Pi_{2}^{1 / 6} \cdot \Pi_{3}^{1 / 2}$,

where $c_{\text {Hunt }}=\Gamma \cdot 2^{1 / 6} \cdot(\sin \varphi)^{1 / 3}$.

\section{b. Schumann Hypothesis}

Schumann [36] ran a large-eddy simulation (LES) of the atmosphere above an unbounded, inclined, rough plane for constant and uniform heat flux and a linearly stratified background at rest. From the LES output Schumann determined the steady state maximum upslope flow velocities. Applying values typical of the Minnekhada site (slope angle of $19^{\circ}$ and momentum roughness lengths ranging from $0.1 \mathrm{~m}$ to $1.0 \mathrm{~m}$ ), Schumann's LES output gives

$U_{\text {max }, a} \approx 2.1\left(g \beta_{a} \frac{Q_{H, a}}{N_{a}}\right)^{1 / 2}$.

The field observations at Minnekhada Park never reached a steady state, as assumed in (8), before the onset of largerscale flows roughly around noon contaminated slope flow observations. However, already at 1100 PST (Pacific Standard Time) on 25 July 2001 (from hereon called the 'test case', Table 4), the observed maximum upslope flow velocity of $3.8 \pm 0.5 \mathrm{~m} \mathrm{~s}^{-1}$ greatly exceeded the steady state value $1.5 \mathrm{~m} \mathrm{~s}^{-1}$ predicted by (8). Therefore, the 'Schumann hypothesis' is defined for atmosphere and water tank by replacing the empirical factor 2.1 in (8) with an unknown coefficient $c_{S c h u}$, and using the definition of $\Pi_{3}$ in Table 2:

$U_{S c h u} * \equiv c_{S c h u} \cdot \Pi_{3}^{1 / 2}$.

\section{c .Chen Hypothesis}

Chen et al. [26] derived an upslope flow velocity scale for atmosphere and water tank from a balance between horizontal advection and pressure gradient term. Assuming again an encroachment model and using (4), a ND modification that allows for friction is given by,

$U_{\text {Chen }} *=c_{\text {Chen }} \cdot \Pi_{2}^{1 / 2} \cdot \Pi_{3}^{1 / 2}$,

where $c_{\text {Chen }}=1$ without friction. Further assumptions underlying (10) are:

(i) $h \leq H$;

(ii) $\mathrm{CBL}$ and upslope flow layer are identical;

(iii) CBL depth is equal over plain and slope;

(iv) the environmental stratification above the upslope flow layer is undisturbed; and

(v) the maximum upslope flow velocity occurs at the ridge top.

\section{d. Gravity Current Hypothesis}

Generalizing $U_{\text {Chen }}=U$ and $h=d$ in (10) and multiplying with $H$ and $N$, gives

$U=c_{\text {Chen }}\left(g_{s} d\right)^{1 / 2}$,

where

$g_{s} \equiv\left\{\begin{array}{cl}g \beta_{a} \theta_{s, a} & \text { for atmosphere } \\ \frac{g}{\alpha_{0, w}} \alpha_{s, w} & \text { for water tank }\end{array}\right.$

is the reduced buoyancy scale; $\theta_{s, a}, \alpha_{s, w}$ and $\alpha_{0, w}$ as before. In the water tank, the upslope flow depth decreases over the slope towards the ridge height, and a plain-plateau flow at ridge height seems to act as a lid [29]. Thus, we suggest an alternative hypothesis,

$U=c_{\text {Grav }}\left(g_{s} H\right)^{1 / 2}$,

where $H$ is the height of the ridge. It is interesting to note that this form of dependence is the same as for a gravity current flowing into a fluid of steadily decreasing depth at a given point, say the mid-point of the slope [37]. Of course, the geometry here is different as the current is actually on the slope. However, the variation in speed of a gravity current on a slope is a weak function of the slope [38] and will appear in a dependence on $\Pi_{1}$. Thus, we will refer to this hypothesis as the gravity current hypothesis. As above, applying an encroachment model giving (4) and using the definitions of the $\mathrm{Pi}$ groups in Table 2, this alternative hypothesis can be defined for atmosphere and water tank by,

$U_{\text {Grav }} *=c_{\text {Grav }} \cdot \Pi_{2}^{1 / 4} \cdot \Pi_{3}^{1 / 4}$.

Of all four hypotheses introduced in this section, only the gravity-current hypothesis gives a dimensional maximum upslope flow velocity that depends on the ridge height $H$, because $\Pi_{2}$ is independent of $H$ and $\Pi_{3}^{1 / 2} \propto H^{-1}$, which cancels after multiplying (7), (9), and (10) by $H$ and $N$. Therefore, only in the gravity-current hypothesis, the upslope flow velocity over any point of the slope depends on the height of the ridge that may be many kilometers (in the atmosphere) or tens of centimeters (in the tank) away and in that sense is the only one of the four hypothesis that treats upslope flows as non-local phenomena. The hypotheses are summarized in Table $\mathbf{3}$.

\section{RESULTS}

In part I it was demonstrated that ND CBL depths in atmosphere and water tank were equal while ND upslope flow velocities were substantially different. The analysis was limited to particular points in time. Here the investigation will be much extended based on the four hypotheses introduced in the previous section.

If ND upslope flow velocities in atmosphere and water tank are functions of $\Pi_{1}, \Pi_{2}$, and $\Pi_{3}$, only, then the 
hypotheses must hold for both systems. In particular, in a monomial of form

$U^{*}=c \cdot \prod_{2}^{m_{1}} \cdot \prod_{3}^{m_{2}}$

the constants $m_{1}, m_{2}$, and $c$ must be equal for atmosphere and water tank. Note that the aspect ratio $\Pi_{1}$ is constant and equal in atmosphere and tank and therefore included in the constant $c$. Bayesian analysis is used to determine the joint probability density functions (PDF) of the exponents $m_{1}$ and $m_{2}$ given the data $D$. After marginalizing, i.e. integrating, over $c$ (see Appendix)

$p\left(m_{1}, m_{2} \mid D\right) \propto \sum_{k=0}^{k_{H}}\left[\sum_{i=1}^{n}\left(U^{*}{ }_{i}-c_{L} \cdot 10^{k / n_{s}} \cdot \prod_{2, i}^{m_{1}} \cdot \prod_{3, i}^{m_{2}}\right)^{2}\right]^{-n / 2}$,

where $k_{H}=n_{s} \log _{10}\left(c_{H} / c_{L}\right), n_{s}$ is the number of steps per order of magnitude in the range of $c$ from a lowest value $c_{L}$ to a highest value $c_{H}$, and $n$ is the number of data points.

Table 3. Summary of Hypotheses for ND Upslope Flow Velocities

\begin{tabular}{|c|c|}
\hline Hypothesis & Equation \\
\hline \hline Hunt & $U_{\text {Hutu }}{ }^{*}=c_{\text {Hutt }} \cdot \Pi_{2}^{1 / 6} \cdot \Pi_{3}^{1 / 2}$ \\
\hline Schumann & $U_{\text {Schu }} * c_{\text {Schu }} \cdot \Pi_{3}^{1 / 2}$ \\
\hline Chen & $U_{\text {Chen }}{ }^{*}=c_{\text {Chen }} \cdot \Pi_{2}^{1 / 2} \cdot \Pi_{3}^{1 / 2}$ \\
\hline Gravity-current & $U_{\text {Grav }} *=c_{\text {Grav }} \cdot \Pi_{2}^{1 / 4} \cdot \Pi_{3}^{1 / 4}$ \\
\hline
\end{tabular}

In the mornings of 25 and 26 July 2001, $n=23$ vertical profiles of wind speed were measured. For each profile $i$, the corresponding $\Pi_{2, i}$ and $\Pi_{3, i}$ were estimated and the ND maximum velocity $U^{*}{ }_{i}$ calculated. The contour plot of the PDF on the lhs of (16) is elliptic with a large ratio between major and minor axes and an almost $45^{\circ}$ tilt relative to the Cartesian axes (Fig. 2a). The reason for the strong tilt and ellipticity is a strong correlation between $\Pi_{2, a}$ and $\Pi_{3, a}$ (correlation coefficient 0.94 ), because both Pi groups depend on time $t_{a}$ via $Q_{H, a}$ and $E_{a}$. It is possible to transform $\Pi_{2, a}$ and $\Pi_{3, a}$ into two approximately decoupled Pi groups $\Pi_{2, a}$ and $\Pi_{3, a}{ }^{\prime}=\Pi_{3, a} / \Pi_{2, a} \quad$ (correlation coefficient -0.24 ), because $\Pi_{3, a}{ }^{\prime}$ only weakly depends on $t_{a}$ for the morning hours used in the analysis [39]. Our field observations for the new Pi group $\Pi_{3, a}{ }^{\prime}$ cover only a narrow range from $2.5 \times 10^{-5}$ to $4.1 \times 10^{-5}$. This narrow range is the main reason for the large uncertainty of the joint PDF $p\left(m_{1}, m_{2} \mid D\right)$ in Fig. (2a) (large contour areas represent large uncertainty), besides measurement uncertainties and natural variability. To provide enough observational evidence for or against the various upslope flow velocity hypotheses, a wide range of values for $\Pi_{3, a}{ }^{\prime}$ is needed. Because $\Pi_{3, a}{ }^{\prime}$ depends on maximum surface heat flux, stability, and only weakly on $t_{a}$, a wide range of $\Pi_{3, a}$ ' requires observations to be carried out over several days with different background stabilities or at different times of year to achieve substantial differences in maximum surface heat flux.

The representation of $p\left(m_{1}, m_{2} \mid D\right)$ in Fig. (2a) is more informative than individual confidence intervals of the exponents $m_{1}$ and $m_{2}$, which envelope the corresponding contour lines. For example, the $95 \%$ confidence intervals of $m_{1}$ and $m_{2}$ in Fig. (2a) include the Chen hypothesis, and based on these confidence intervals the Chen hypothesis cannot be rejected. However, because the Chen hypothesis lies outside of the 0.05 contour line, i.e. the $95 \%$ credible region, of the joint PDF of $m_{1}$ and $m_{2}$, the Chen hypothesis can be rejected. On the other hand, the Hunt, Schumann, and gravity-current hypotheses lie well within the $95 \%$ credible region and can therefore not be rejected by the field data.

Having greater control over the water-tank parameters and better and more measurements, results in a much narrower joint PDF for the water-tank (Fig. 2b). Because heat flux was held steady in most tank experiments, $\Pi_{3, w}$ depends only weakly on time. Consequently, $\Pi_{2, w}$ and $\Pi_{3, w}$ are only weakly correlated and the ellipse in Fig. (2b) is only slightly stretched and tilted. A direct comparison of the joint PDF $p\left(m_{1}, m_{2} \mid D\right)$ for atmosphere and water tank (Fig. 2a) reveals that they are substantially different (with more than $95 \%$ probability). Hence, there is sufficient evidence in the field and tank data that ND upslope flow velocities have different functional relationships with $\Pi_{2}$ and $\Pi_{3}$. Moreover, all four hypotheses introduced in section 3 are far outside the 0.05 contour line of the tank PDF $p\left(m_{1}, m_{2} \mid D\right)$. Because $\Pi_{1}$ is equal for atmosphere and tank and any dependence on $\Pi_{2}$ and $\Pi_{3}$ was already accounted for in (15), the coefficients $c$ must depend on Pi groups other than $\Pi_{1}, \Pi_{2}$, and $\Pi_{3}$. In the following discussion section, evidence is provided for fluid-dynamically smooth tank flow. It will be determined how this leads to different functional dependence of $U^{*}$ on $\Pi_{2}$ and $\Pi_{3}$ in the water tank. Possible dependencies on other Pi groups will be briefly discussed.

\section{DISCUSSION}

\section{a. Dependence on Momentum Roughness Length}

The Hunt hypothesis contains a very weak dependence of $U^{*}$ on ND momentum roughness length $\Pi_{10}$ through (6). Schumann [36] also found in his LES model runs a very weak dependence on ND momentum roughness length:

$U_{a} * \propto \Pi_{10, a}^{-0.033} \cdot \Pi_{3, a}^{1 / 2}$ 

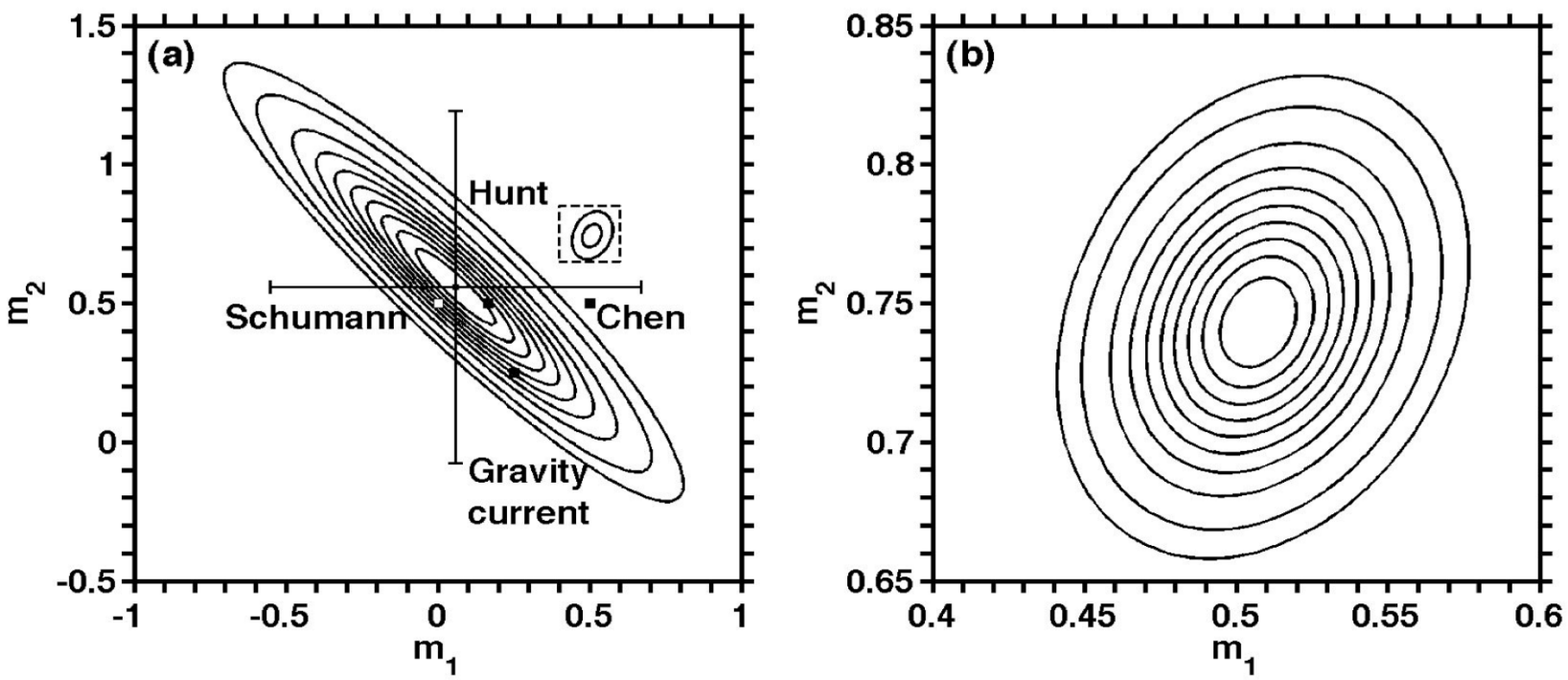

Fig. (2). Joint PDF $p\left(m_{1}, m_{2} \mid D\right)$ of the exponents $m_{1}$ and $m_{2}$ in an empirical relationship $U^{*}=c \cdot \Pi_{2}^{m_{1}} \cdot \Pi_{3}^{m_{2}}$ between ND upslope flow velocity $U^{*}$ and the two Pi groups $\Pi_{2}$ and $\Pi_{3}$. For the large ellipses in (a) and (b), contour lines are shown for 0.05 (outer line) and from 0.1 to 0.9 in steps of 0.1. (a) Large ellipse is for the field data on the mornings of 25 and 26 July 2001; small ellipse (only the 0.05 and 0.5 contour lines are shown) is for the data from seven water-tank experiments (Table 1); labeled squares are the positions of the upslope flow velocity hypotheses discussed in section 3; error bars indicate $95 \%$ confidence intervals from a nonlinear regression. (b) Zoomed into the dashed region in (a) around the PDF for the tank data.

for a slope angle of $10^{\circ}$. Schumann's one-dimensional slope with the assumption of a steady state may not be a good representation of the field and water-tank observations used here, but it is worth pursuing the idea of a functional dependence of $U^{*}$ on $\Pi_{10}$ further. Assume a dependence on $\Pi_{10}$ was contained in the coefficients $c$ in the four hypotheses introduced in section 3. Separating this dependence from the coefficient generalizes (15):

$U^{*}=c_{1} \cdot \Pi_{10}^{-A} \cdot \Pi_{2}^{m_{1}} \cdot \Pi_{3}^{m_{2}}$,

where a minus sign was added to the exponent of $\Pi_{10}$ because it can be expected that $U^{*}$ is inversely related to $\Pi_{10}$ so that the unknown parameter $A>0$.

For the atmosphere, all but the Chen hypothesis give functional dependences of $U^{*}$ on $\Pi_{2}$ and $\Pi_{3}$ that agree with field observations. Hence, the field observations support an approximately constant momentum roughness length in the atmosphere, as would be expected from landuse characteristics at the field site. For the water-tank observations, however, a hypothesis of form (18) requires that ND momentum roughness length $\Pi_{10, w}$ is a function of at least one of the two Pi groups $\Pi_{2, w}$ and $\Pi_{3, w}$. A hypothesis for such dependence will be presented next.

\section{a. Similarity Violation as a Result of Fluid-Dynamic Feedback}

In wind tunnel experiments, aerodynamical roughness is an important, yet often violated, scaling requirement [40]. In general, a flow is fluid-dynamically smooth if the viscous sublayer of depth $\delta_{w} \approx \frac{5 v_{w}}{u_{*, w}}$

(chapter XX in [41]) is deeper than surface roughness protuberances. In the atmosphere over land surfaces, this is practically never the case [31]. However, an order-ofmagnitude estimation shows that in our tank experiments the flow was fluid-dynamically smooth: The ratio between velocity and friction velocity is of order 10, i.e. $U_{w} / u_{*, w} \sim 10$ [41, 42]; with the tank values for $U_{w}$ and $v_{w}$ in Table 4 this gives $\delta_{w} \sim 0.01 \mathrm{~m}$, which is approximately two orders of magnitude larger than the roughness elements on the well-sanded and painted tank bottom.

Because of fluid-dynamical smoothness, momentum roughness length in the water tank is not a function of distribution and size of roughness elements but of viscosity and friction velocity (Chapter 7 in [42]):

$z_{m, w} \approx \frac{0.11 v_{w}}{u_{*, w}}$.

An increase of upslope flow velocity leads to increased friction velocity, which in turn leads to decreased roughness length in (20). This permits further increase of upslope flow velocity. A possible approach to quantifying this positive fluid-dynamical feedback will be presented next.

\section{b. Quantification of Fluid-Dynamic Feedback}

The goal is to express $U_{w}{ }^{*}$ as a monomial of the two Pi groups $\Pi_{2, w}$ and $\Pi_{3, w}$ by substituting (20) into the definition of $\Pi_{10, w}$ in Table 4, which is substituted into (18) to give 
$U_{w} * \approx c_{1} \cdot\left(\frac{0.11 v_{w}}{u_{*, w} H_{w}}\right)^{-A} \cdot \Pi_{2}^{m_{1}} \cdot \Pi_{3}^{m_{2}}$

To express the friction velocity $u_{*, w}$ in terms of known quantities, we use convective transport theory [43],

$u_{*}=\left(C_{* D} w_{*} U\right)^{1 / 2}$,

where the momentum transport coefficient $C_{*_{D}}$ depends on the surface characteristics, $U$ is the maximum in the vertical profile of upslope flow velocity, and

$w_{*}=\left(g \beta h Q_{H}\right)^{1 / 3}$

is the convective velocity scale.

Table 4. Data for Test Case

\begin{tabular}{|c|c|c|c|}
\hline Name & Symbol (Units) & Atmosphere & Water Tank \\
\hline \hline Ridge height & $H(\mathrm{~m})$ & 760 & 0.149 \\
\hline $\begin{array}{c}\text { Instantaneous } \\
\text { heat flux }\end{array}$ & $Q_{H}\left(\mathrm{~K} \mathrm{~m} \mathrm{~s}^{-1}\right)$ & $0.21 \pm 0.05$ & $(1.9 \pm 0.2) \times 10^{-3}$ \\
\hline Energy density & $E\left(\mathrm{~K} \mathrm{~m}^{-3}\right.$ & $1600 \pm 400$ & $0.56 \pm 0.05$ \\
\hline Buoyancy frequency & $N\left(\mathrm{~s}^{-1}\right)$ & $0.015 \pm 0.001$ & $0.38 \pm 0.02$ \\
\hline Buoyancy parameter & $g \beta\left(\mathrm{m} \mathrm{s}^{-2} \mathrm{~K}^{-1}\right)$ & 0.036 & 0.0026 \\
\hline $\left.\begin{array}{c}\text { Kinematic viscosity } \\
\hline \begin{array}{c}\text { Prandtl number } \\
\text { at } 25^{\circ} \mathrm{C}\end{array}\end{array} \mathrm{m}^{-1}\right)$ & $1.52 \times 10^{-5}$ & $8.9 \times 10^{-7}$ \\
\hline $\begin{array}{c}\text { CBL depth } \\
\mathrm{Pr}^{-1}\end{array}$ & 0.72 & 6.1 \\
\hline $\begin{array}{c}\text { Upslope flow } \\
\text { velocity }\end{array}$ & $U(\mathrm{~m} \mathrm{~s})$ & $720 \pm 120$ & $0.14 \pm 0.02$ \\
\hline
\end{tabular}

Atmospheric data are for the field site at Minnekhada Park at 1100 PDT 25 July 2001; water-tank experiment was designed such that, i.e. $\Pi_{2, w}=\Pi_{2, a}$ and $\Pi_{3, w}=\Pi_{3, a}$. Uncertainties roughly represent $95 \%$ confidence intervals.

Equation (22) was extensively validated for atmospheric convection over land and ocean surfaces [43-45]. The derivation of (22) in [43] applies equally to free convection in air or water. It assumes that surface- and micro-layer processes can be ignored, therefore it can also be applied to fluid-dynamically smooth flow. To ignore these processes, they must be fast enough to maintain higher temperatures in surface- and micro-layer than in the mixed layer. This was the case in all tank experiments, because convection and upslope flows continued for at least another minute after heat flux was shut down. Stull [43] showed that (22) also holds under non-calm conditions as long as the mixed-layer Richardson number

$R_{*} \equiv\left(\frac{w_{B}}{U}\right)^{2}$,

is greater than 3 ('quasi-free convection'). Here, the buoyancy velocity scale $w_{B}$ is defined by
$w_{B}^{2} \equiv\left\{\begin{array}{cc}g \beta_{a} \Delta \theta_{\text {skin,a }} h_{a} & \text { for atmosphere } \\ \frac{\mathrm{g}}{\alpha_{0, \mathrm{w}}} \Delta \alpha_{s k i n, w} \mathrm{~h}_{\mathrm{w}} & \text { for water tank, }\end{array}\right.$

where $\Delta \theta_{\text {skin,a }}$ and $\Delta \alpha_{\text {skin,w }}$ are the differences of potential temperature at the land surface and specific volume at the tank bottom to the corresponding quantities in the mixed layer. All other quantities were introduced before, and typical values used here are given in Table 4. Because we do not have measurements of the skin temperatures, we estimate lower limits. At the field site we measured differences between 2-m and mixed-layer temperatures of approximately $2 \mathrm{~K}$. Because skin temperatures can be expected to be substantially larger, we use $\Delta \theta_{\text {skin, } a}=3 \mathrm{~K}$ as a lower limit, which was also the lowest value observed in Oklahoma under much weaker surface heat fluxes [43]. Substituting this value into (25) gives $w_{B, a}>9 \mathrm{~m} \mathrm{~s}^{-1}$, and the mixed-layer Richardson number becomes $R_{*, a}>5$, meeting the requirement for quasi-free convection. In the water tank, rising thermals hitting the $\mathrm{CT}$ probes lead to rapid increases in specific volume, which are used here as a conservatively low estimate of the difference between skin and mixed-layer temperatures: $\Delta \alpha_{\text {skin,w }}=0.5 \times 10^{-6} \mathrm{~m}^{3} \mathrm{~kg}^{-1}$ (Fig. 7 in [29]). This value gives $w_{B, w}>0.02 \mathrm{~m} \mathrm{~s}^{-1}$ and $R_{*, w}>16$, indicative of quasi-free convection.

The convective boundary-layer depth $h$ is substituted from the encroachment model (4) to give the convective velocity scale (23). Then the convective velocity scale (23) is substituted into (22) to give the friction velocity, which is substituted into (21) to give

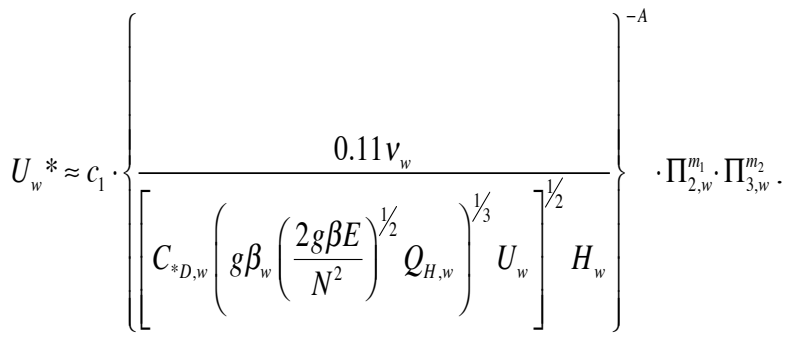

On the rhs of the last equation, the viscosity $v_{w}$ appears, so that ND upslope flow velocity depends on ND viscosity $\Pi_{4, w}$. Moreover, the appearance of upslope flow velocity $U_{w}$ with the positive exponent $A / 2$ on the rhs quantifies the positive feedback mechanism described qualitatively in the previous subsection. Expanding the rhs with monomials of $H_{w}$ and $N_{w}$, applying the definitions of $\Pi_{2, w}, \Pi_{3, w}$, and $\Pi_{4, w}$ in Table 2, and solving for $U_{w}{ }^{*}$ yields

$U_{w} * \approx c_{1}^{\frac{2}{2-A}} \cdot 0.11^{\frac{-2 A}{2-A}} \cdot 2^{\frac{A}{6(2-A)}} \cdot C_{* D, w}^{\frac{A}{2-A}} \cdot \prod_{4, w}^{\frac{-2 A}{2-A}} \cdot \prod_{2, w}^{\frac{A+12 m_{1}}{6(2-A)}} \cdot \prod_{3, w}^{\frac{A+4 m_{2}}{2(2-A)}}$.

The first three factors are constant. The fourth and fifth factors are not constant, but the tight contour lines in Fig. (2) suggest that they did not vary much. This will be further 
discussed below. The pairs of exponents $\left(\frac{A+12 m_{1}}{6(2-A)}, \frac{A+4 m_{2}}{2(2-A)}\right)$ are a parametric representation of a path in the $m_{1} \times m_{2}$ plane. Each point on the path corresponds to a particular value of the exponent $A$ of ND momentum roughness length $\Pi_{10, w}$ in (18). Most probable values of $A$ are those within the PDF contours near the mode of $p\left(m_{1}, m_{2} \mid D\right)$. There are four different paths, one for each hypothesis introduced in section 3 and its fixed pair $\left(m_{1}, m_{2}\right)$ of exponents (Fig. 3a). Only the path for the gravity-current hypothesis is within the $5 \%$ contour of the PDF and runs close to the mode (Fig. $3 \mathbf{b}$ ). There is a range of probable values for $A$. Representative values of simple rationals for $A$ are shown in Fig. (3b). We chose for the following discussion $A=3 / 4$ fairly close to the mode, because it seems more likely that this value could be derived from first principles than $4 / 5$ or $5 / 6$. The gravity-current hypothesis therefore agrees well with water-tank observations if the ND momentum roughness length with an exponent of $A=3 / 4$ is included in (27):

$U_{w} * \approx 15.15 c_{1}^{8 / 5} \cdot C_{* D, w}^{3 / 5} \Pi_{4, w}^{-6 / 5} \cdot \Pi_{2, w}^{1 / 2} \cdot \Pi_{3, w}^{7 / 10}=c_{2, w} \cdot \Pi_{2, w}^{1 / 2} \cdot \Pi_{3, w}^{7 / 10}$.

Substituting $A=3 / 4$ into (18) gives the corresponding equation for the atmosphere:

$U_{a} *=c_{1} \cdot \Pi_{10, a}^{-3 / 4} \cdot \Pi_{2, a}^{1 / 4} \cdot \Pi_{3, a}^{1 / 4}=c_{2, a} \cdot \Pi_{2, a}^{1 / 4} \cdot \Pi_{3, a}^{1 / 4}$.

Here $c_{2, a}$ depends on surface characteristics and slope angle, but is constant with respect to the quantities changed

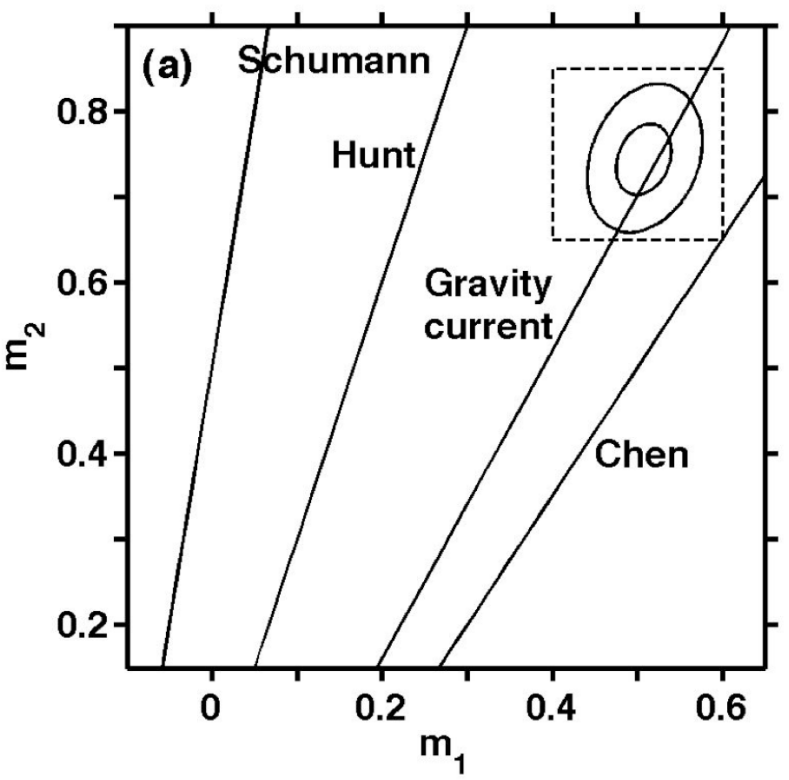

in our experiments listed in Table 1: $N_{w}, Q_{H, w}$, and $E_{w}$. In the water tank, $c_{2, w}$ depends rather strongly on $N_{w}$ : $c_{2, w} \propto N_{w}^{6 / 5}$. However, because $U_{w} * \propto N_{w}^{-2 / 5} \cdot E_{w}^{1 / 2} \cdot Q_{H, w}^{1 / 5}$ and the range of buoyancy frequency $N_{w}$ and instantaneous heat flux $Q_{H, w}$ in our tank experiments is rather small (Table 1), the posterior distribution of $U_{w}{ }^{*}$ in Figs. $(2,3)$ is dominated by $E_{w}$, which ranges from 0 to its maximum value. More experiments over a much wider range of buoyancy frequencies $N_{w}$ will be required in the future to test the dependence of ND upslope flow velocity on $N_{w}$ and therefore test the applicability of convective transport theory to upslope flows in water tanks.

\section{c. Plausibility Test and Future Research Needs}

From the data of the test case in Table $4, U_{a}^{*}=0.33$ and $U_{w} *=0.088$. It seems counterintuitive at first that a fluiddynamically smooth flow is slower than a rough flow. Estimating roughly $z_{m, a} \approx 0.3 \mathrm{~m}$ at the field site, the momentum roughness length in the tank that is required from similarity with the atmosphere, $\Pi_{10, w}=\Pi_{10, a}$, is $z_{m, w}=z_{m, a} H_{w} / H_{a} \approx 0.06 \mathrm{~mm}$. In other words, if the flow in the tank was fluid-dynamically rough with a roughness length of $0.06 \mathrm{~mm}$ then similarity of ND upslope flow velocity could be expected. However, the smooth flow in the tank has the characteristics of a rough flow over a surface with much larger momentum roughness length: Field observations for the test case (Table 4) substituted into (29)

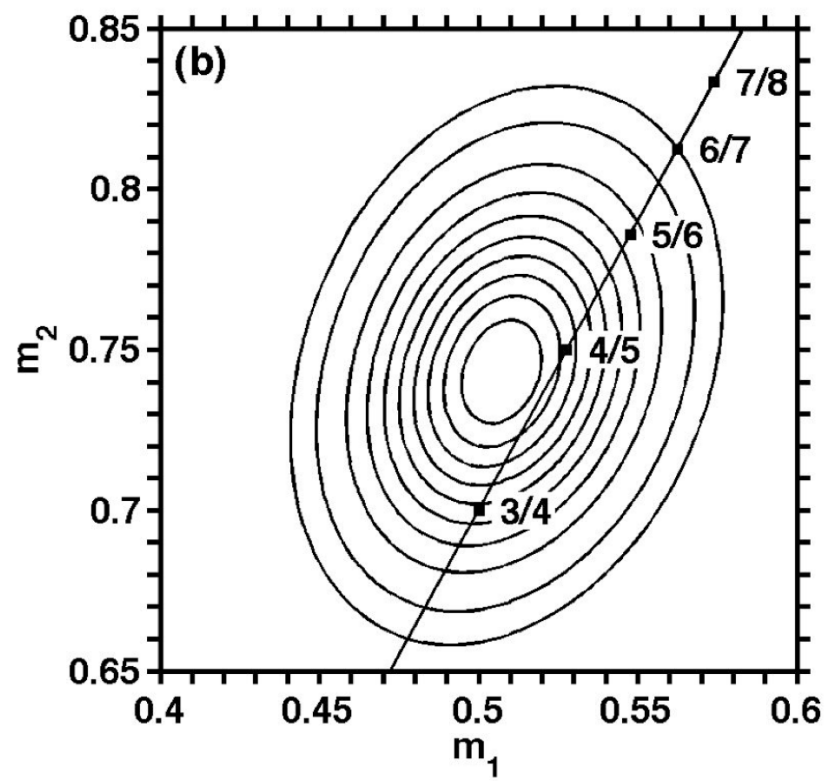

Fig. (3). Parameter paths of the exponent $A$ in upslope flow velocity hypotheses of form $U_{w} *=c_{1} \cdot \Pi_{10, w}^{-A} \cdot \Pi_{2, w}^{m_{1}} \cdot \Pi_{3, w}^{m_{2}}$, superimposed on the joint PDF of $m_{1}$ and $m_{2}$ for the tank observations, only. The pairs $\left(\frac{A+12 m_{1}}{6(2-A)}, \frac{A+4 m_{2}}{2(2-A)}\right)$ are shown for four upslope flow velocity hypotheses as labeled in (a). Contour plots of the PDF are shown as in Fig (2). (b) is zoomed into the dotted rectangular region of (a). Representative values of $A$ are shown in (b) as labeled squares. 
give $c_{1} \approx 1.1 \times 10^{-3}$. A corresponding rough flow in the water tank should follow the equivalent equation to (29),

$U_{w}{ }^{*}=c_{1} \cdot \Pi_{10, w}^{-3 / 4} \cdot \Pi_{2, w}^{1 / 4} \cdot \Pi_{3, w}^{1 / 4}$,

with the same value for $c_{1}$ as in the atmosphere. Substituting this value and the tank observations of the test case (Table 4) into (30) gives $z_{m, w} \approx 0.3 \mathrm{~mm}$. In other words, with respect to momentum roughness length, the smooth flow in the water tank represents a rough atmospheric flow over a surface with a roughness length about five times greater than the one estimated for the field site. Therefore, from (29), the ND upslope flow velocity in the atmosphere should be $5^{3 / 4}$ times the tank value, in good agreement with the data above.

The dependence of the gravity-current hypothesis on roughness length as detailed here can only be tentative and guide in a search for underlying first principles. Future research will have to address several questions and challenges: Is convective transport theory applicable to smooth flows? Can the unknown coefficients $c_{1}$ and $C_{*_{D, w}}$ in (28) be derived from first principles? Moreover, it is desirable to refine the upslope flow velocity hypotheses by replacing the encroachment model with the entrainment model. Finally, with more tank data the probability density function of $m_{3}$ in an upslope flow hypothesis of form

$U_{w}=c_{w} \cdot \Pi_{4, w}^{m_{3}} \cdot \Pi_{2, w}^{m_{1}} \cdot \Pi_{3, w}^{m_{2}}$

can be determined and compared with $m_{3}=-6 / 5$ in (28).

Such future research would make it possible to quantitatively scale between water tank and atmosphere, albeit not simply by applying (15) with the constants $m_{1}$, $m_{2}$, and $c$ being equal for atmosphere and water tank. Currently, even if fluid-dynamical smoothness could be overcome in water-tank experiments, the constants $m_{1}, m_{2}$, and $c$ in (15) remain poorly constrained. Particularly, more accurate field data for nearly idealized environmental settings are needed. This paper suggests that previously observed quantitative agreement was within the uncertainties associated with field and tank measurements and with environmental settings that were either badly known or difficult to model in the water tank. However, previous research mentioned in the introduction has demonstrated the value of water-tank experiments to qualitatively model atmospheric flows over sloping terrain. For example, Reuten et al. (2007) demonstrated similar development of pollutant layers in water tank and field observations.

\section{d. The Role of Other Pi Groups}

The previous subsections of the discussion provide a tentative explanation for the different functional dependences of ND upslope flow velocities in tank and atmosphere on the governing parameters. The explanation focused on the importance of momentum roughness length $\Pi_{10}$ in the atmosphere and led to the inclusion of ND viscosity $\Pi_{4}$ in the water tank. A brief discussion of the potential role of other ND governing parameters is provided next.

\section{Thermal Roughness Length and Diffusivity}

A hint at another important difference between tank and atmosphere comes from [27], who included the thermal roughness length $z_{T}$, (5) and (6). The ratio between momentum and thermal roughness length strongly depends on the Prandtl number (which is very different for air and water, Table 4) and surface properties. For the field site, $z_{m, a} \gg z_{T, a}$ [31], and for smooth tank flow $z_{m, w} \gg z_{T, w}$ by many orders of magnitude (table 4.1 in [46]). Hence, heat transport is very inefficient in both systems. Further research into the coefficient $\Gamma_{a}$ for the atmosphere in [27], (6), and $\Gamma_{w}$ for the water tank is required to assess the impact of a similarity violation of ND thermal roughness length $\Pi_{11}=z_{T} / H$ on upslope flow velocity in the Hunt hypothesis. It was shown above that the characteristics of fluid-dynamically smooth flow introduce a dependence on ND viscosity $\Pi_{4, w}$ in (28). Future research will have to investigate if a dependence of ND upslope flow velocity on ND thermal roughness length $\Pi_{11}$ introduces a dependence on ND thermal diffusivity $\Pi_{5, w}$ in the water tank.

\section{Reynolds Number}

As the flow is dependent on the viscosity, it is Reynoldsnumber (Re) dependent. A more direct and usual route would be to investigate the effect of Reynolds number directly, rather than consider roughness lengths. However, as was pointed out in part I, this approach is inconclusive. The critical Reynolds number, above which the flow would be dominated by turbulence, is strongly dependent on the choice of length and velocity scales and the particular system and flow geometry. Using the definition in [26], the tank experiments used in this part give $\operatorname{Re}=L U / v=(2.2-8.7) \times 10^{3}$. According to [26], this would suggest similarity with the atmosphere based on a critical pipe flow Reynolds number. However, the geometry in the water tank is not that of a pipe flow and may therefore have a different critical value. An example of a different geometry (a plume stack) is given by Contini et al. (2009) where they find that a value of 2196 in a wind tunnel is insufficient to achieve similarity with the full-scale atmosphere. In contrast [39] defined the Reynolds number based on upslope-flow profiles observed in the tank assuming the geometry of an open channel flow: Only for the fastest flows observed in our experiments, values in the range 1350-1800 of intermittently turbulent flow are reached. The approach taken in this study via the momentum roughness lengths provides deeper insight into the flow characteristics in the tank.

\section{Plain and Plateau Lengths}

Non-dimensional plain and plateau lengths, $\Pi_{6, w}$ and $\Pi_{7, w}$, cannot be significant for the similarity violation. The water-tank experiments (Table 1) had substantially different 
$\Pi_{6, w}$ and $\Pi_{7, w}$ but $p\left(m_{1}, m_{2} \mid D\right)$ is very narrow.

Furthermore, $p\left(m_{1}, m_{2} \mid D\right)$ for all seven experiments is narrower than for any subset of six experiments (not shown). In other words, each tank experiment contributes to improved predictions of $m_{1}$ and $m_{2}$ even if inclusion of the data increases the range of $\Pi_{6, w}$ and $\Pi_{7, w}$.

\section{Tank Width}

Observations early into the tank experiments show no influence of friction at the lateral side walls of the tank on upslope flow velocities near the slope centre. At later times, which we did not use for the analyses here, more complex 3D flow structures developed which can be attributed to temperature gradients and possibly friction at the lateral side walls (figure 8 in [29]). The opposite effect, lateral side walls suppressing horizontally mass-compensating flows and forcing return flows against gravity above the upslope flow, seems plausible. However, despite a lack of lateral confinement at the field site [30] observed flows in downslope direction above the upslope flows, which compensated most of the upslope mass flux. Therefore, ND upslope flow velocities should not substantially depend on ND tank width $\Pi_{8, w}$.

\section{Water Depth and Gravity Waves}

Non-dimensional water depth over the plain, $\Pi_{9, w}$, is unlikely an important factor for upslope flow velocities, because in all experiments the water surface was far above the top of the CBL. In agreement with [25], we observed elevated layers of alternating flows above the plain-plateau flow. These were shallow, their velocities decreased substantially with height, and they never seemed to affect water near the top surface.

An indirect effect of the confinement of the tank by $\Pi_{6, w}$ to $\Pi_{9, w}$ could be that energy dissipation by gravity waves in the atmosphere is greater than in the water tank, but that contradicts observations of greater upslope flow velocities in the atmosphere (part I). In can be concluded that it is unlikely that ND upslope flow velocities depend substantially on $\Pi_{6, w}$ to $\Pi_{9, w}$ and that such a dependence could explain the similarity violation.

\section{CONCLUSIONS}

Four hypotheses for ND maximum upslope flow velocities were introduced based on $[27,36,26]$, and a gravity-current flow into a fluid of decreasing depth. The hypotheses were all of form $U^{*}=c \cdot \prod_{2}^{m_{1}} \cdot \prod_{3}^{m_{2}}$ with unknown coefficient $c . \Pi_{2}=\frac{E N}{Q_{H}}$ and $\Pi_{3}=\frac{g \beta Q_{H}}{H^{2} N^{3}}$ were initially assumed the only governing ND parameters. The Bayesian joint PDF of the exponents $m_{1}$ and $m_{2}$ differed between atmosphere and water tank with greater than $95 \%$ probability. The Hunt, Schumann, and gravity-current hypotheses agreed well with the PDF for the field data, but none of them agreed with the PDF for the tank data. It was concluded that ND upslope flow velocity requires additional dependence on other Pi groups. Observational evidence rules out $\mathrm{Pi}$ groups related to the size and geometry of the tank $\left(\Pi_{6, w}\right.$ to $\left.\Pi_{9, w}\right)$. Estimations of surface roughness and viscous sublayer depth suggest that the upslope flow in the water tank is fluid-dynamically smooth, and therefore momentum roughness length $z_{m, w}$ depends on friction velocity. This results in a stronger dependence of $U_{w}{ }^{*}$ on $\Pi_{2, w}$ and $\Pi_{3, w}$ in the tank than in the atmosphere, because an increase of $\Pi_{2, w}$ or $\Pi_{3, w}$ leads to a decrease of $z_{m, w}$ and therefore stronger increase of $U_{w}{ }^{*}$ than for fluiddynamically rough flow. It was demonstrated that only for the gravity-current hypothesis the inclusion of ND momentum roughness length in $U_{w}{ }^{*}$ can lead to good agreement with the tank data. This success of the gravitycurrent hypothesis suggests that upslope flows must be treated as non-local phenomena, because only in the gravitycurrent hypothesis are upslope flow velocities dependent on ridge height.

More research should be carried out to test the applicability of convective transport theory to smooth flows, to replace the encroachment model of CBL growth by the more realistic entrainment model, and to test the predicted value $-6 / 5$ of the exponent of ND viscosity $\Pi_{4, w}$ in (28).

The work presented here implies that water-tank models of other thermally driven meso-scale circulations like upvalley flows, sea breezes, and urban heat islands may suffer the same velocity similarity violation as upslope systems. It is difficult in a laboratory setting to ensure both fluiddynamically rough flow and a match between roughness elements in the atmosphere and tank. However, as shown here, it is possible to explicitly include the dependence on the momentum roughness length and use the tank data to distinguish between flow dependence models for the atmosphere.

\section{ACKNOWLEDGEMENTS}

We wish to thank Greg Lawrence for providing laboratory space for the water tank and sharing computer resources and instrumentation. The water tank was built in the workshop of the Department of Civil Engineering at UBC; the work by Bill Leung, Scott Jackson, and Harald Schrempp is gratefully acknowledged. We thank Ian Chan for his help on tank re-design, MatPIV, and several experiments. Funding support was provided by grants from NSERC and CFCAS to Douw Steyn and Susan Allen.

\section{APPENDIX}

\section{Bayesian Parameter Estimation}

This appendix briefly derives the equations used in this communication. Background information and details of the following derivations can be found in [47-50].

Starting point is Bayes' Theorem 
$p(\theta \mid D, I)=\frac{p(\theta \mid I) p(D \mid \theta, I)}{p(D \mid I)}$.

Here, the arguments are propositions and $p$ denotes probabilities of their truths. We follow the common practice to drop the terminology involving 'proposition'; instead we interpret $p(\theta \mid D, I)$ as the joint probability density function (PDF) of the (unknown) model parameters $m_{1}, m_{2}$, and $c$ in (15) and their standard deviation $\sigma$, given the $n$ data $d_{1}, \ldots, d_{n}$ and the background information $I$. Note that $\sigma$ is dominated by unknown environmental noise and therefore must be modeled as an unknown parameter. On the rhs, $p(\theta \mid I)$ is the 'prior probability' assigned to $m_{1}, m_{2}, c$, and $\sigma$ before knowledge of the data; $p(D \mid \theta, I)$ is the 'likelihood' of the data, which is the sampling distribution of the data given particular parameter values $m_{1}, m_{2}, c$, and $\sigma$; finally, the 'global likelihood' $p(D \mid I)$ is a normalization constant. Each of the factors in (1) contains a conditional dependence on the background information $I$, which will be dropped for the remainder of this appendix. Thus, Bayes' theorem (1) becomes

$$
p\left(m_{1}, m_{2}, c, \sigma \mid D\right) \propto p\left(m_{1}, m_{2}, c, \sigma\right) p\left(D \mid m_{1}, m_{2}, c, \sigma\right) .
$$

The likelihood on the rhs is treated first. From the background information, the individual observations can be expressed as

$d_{i}=f_{i}+e_{i}$,

where $f_{i}$ is the model's prediction of the $i^{\text {th }}$ datum; the $e_{i}$ follow a normal distribution with mean zero and standard deviation $\sigma$. Let $D_{i}$ denote the proposition that the $i^{\text {th }}$ datum is $d_{i}$, so that $D=D_{1}, \ldots, D_{n}$. Similarly, define propositions on the errors, such that $E=E_{1}, \ldots, E_{n}$. The sampling distribution of the noise is Gaussian,

$p\left(E_{i} \mid \sigma\right)=\frac{1}{\sqrt{2 \pi} \sigma} \exp \left(-\frac{e_{i}^{2}}{2 \sigma^{2}}\right)$.

Substituting $e_{i}$ from (3) gives the sampling distribution of the data

$$
p\left(D_{i} \mid \theta\right)=\frac{1}{\sqrt{2 \pi} \sigma} \exp \left[-\frac{\left(d_{i}-f_{i}\right)^{2}}{2 \sigma^{2}}\right],
$$

which depends on all parameters $\theta$ and $\sigma$ and the background information $I$. Because the individual observations are independent of each other and the noise is independent and identically distributed, the product rule of probability theory implies,

$$
p\left(D_{1}, \ldots, D_{n} \mid \theta\right)=\prod_{i=1}^{n} p\left(D_{i} \mid \theta\right) .
$$

Substituting (5) into (6) gives

$$
p(D \mid \theta, \sigma)=\frac{1}{(2 \pi)^{n / 2} \sigma^{n}} \exp \left[-\frac{1}{2 \sigma^{2}} \sum_{i=1}^{n}\left(d_{i}-f_{i}\right)^{2}\right] .
$$

Applied to the data and hypotheses in this paper the last equation becomes

$$
p\left(D \mid m_{1}, m_{2}, c, \sigma\right)=\frac{1}{(2 \pi)^{n / 2} \sigma^{n}} \exp \left[-\frac{1}{2 \sigma^{2}} \sum_{i=1}^{n}\left(U^{*}{ }_{i}-c \cdot \Pi_{2, i}^{m_{1}} \cdot \Pi_{3, i}^{m_{2}}\right)^{2}\right],(8)
$$

where $U^{*}{ }_{i}$ are the non-dimensionalized observed upslope flow velocities, $f_{i}=c \cdot \prod_{2, i}^{m_{1}} \cdot \Pi_{3, i}^{m_{2}}$ is the model function evaluated at the values of $\Pi_{2}$ and $\Pi_{3}$ corresponding to each observation $i$, and $m_{1}, m_{2}$, and $c$ are the model parameters.

The first factor on the rhs in (2), the prior probability, can be split using the product rule and assuming that all four parameters $m_{1}, m_{2}, c$, and $\sigma$ are a priori independent of each other,

$p\left(m_{1}, m_{2}, c, \sigma\right)=p\left(m_{1}\right) p\left(m_{2}\right) p(c) p(\sigma)$.

The intention here is to be maximally non-committal about the parameters $m_{1}, m_{2}, c$, and $\sigma$ within sufficiently wide lower and upper boundaries. This can be achieved in two fundamentally different ways [50]. 'Location' parameters can be either positive or negative and usually have a relatively narrow prior range, which applies to $m_{1}$ and $m_{2}$. They are assigned uniform priors

$$
p\left(m_{1}\right)=\frac{1}{m_{1, H}-m_{1, L}} \text { and } p\left(m_{2}\right)=\frac{1}{m_{2, H}-m_{2, L}},
$$

where the subscripts $L$ and $H$ denote lower and upper boundaries, respectively. By contrast, 'scale parameters' are always positive and the lower and upper boundaries often span several orders of magnitude. The standard deviation $\sigma$ and the constant factor $c$ are both treated as scale parameters, which are assigned Jeffreys priors:

$$
p(\sigma)=\frac{1}{\sigma \ln \left(\sigma_{H} / \sigma_{L}\right)} \text { and } p(c)=\frac{1}{c \ln \left(c_{H} / c_{L}\right)} .
$$

Now the rhs of (2) can be determined, but the quantity of interest is $p\left(m_{1}, m_{2} \mid D\right)$, which can be determined from $p\left(m_{1}, m_{2}, c, \sigma \mid D\right)$ by 'marginalizing' over the two parameters $c$ and $\sigma$ :

$p\left(m_{1}, m_{2} \mid D\right)=\int_{\sigma_{L}}^{\sigma_{H}} \int_{c_{L}}^{c_{H}} p\left(m_{1}, m_{2}, c, \sigma \mid D\right) d c d \sigma$.

Substituting (2) and (8)-(11) into (12) gives

$p\left(m_{1}, m_{2} \mid D\right) \propto \int_{c_{L}}^{c_{H}} \frac{1}{c} \int_{\sigma_{L}}^{\sigma_{H}} \frac{1}{\sigma^{n+1}} \exp \left[-\frac{1}{2 \sigma^{2}} \sum_{i=1}^{n}\left(U_{i}^{*}-c \cdot \Pi_{2, i}^{m_{1}} \cdot \Pi_{3, i}^{m_{2}}\right)^{2}\right] d \sigma d c .(13)$

Following the derivation leading to (C.17) in [50] and assuming that the boundaries of integration over $\sigma$ are so 
wide that practically $\sigma_{L} \rightarrow 0$ and $\sigma_{H} \rightarrow \infty$, the inner integral of (13) simplifies so that,

$$
p\left(m_{1}, m_{2} \mid D\right) \propto \int_{c_{L}}^{c_{H}} d c \frac{1}{c}\left[\sum_{i=1}^{n}\left(U_{i}^{*}-c \cdot \Pi_{2, i}^{m_{1}} \cdot \Pi_{3, i}^{m_{2}}\right)^{2}\right]^{-n / 2},
$$

where the gamma function that results from the integration is absorbed in the proportionality.

The integral in (14) is replaced by a sum in which both $c_{k}$ and the step length $\Delta c_{k}$ increase exponentially with index $k$. Let $n_{s}$ denote the number of steps per order of magnitude, then

$$
\begin{aligned}
& c_{k}=c_{L} \cdot 10^{k / n_{s}} \Rightarrow \\
& \Delta c_{k}=c_{k+1}-c_{k}=c_{L} \cdot 10^{(k+1) / n_{s}}-c_{L} \cdot 10^{k / n_{s}}=c_{L} \cdot 10^{k / n_{s}} \cdot\left(10^{1 / n_{s}}-1\right),
\end{aligned}
$$

so that

$$
\frac{\Delta c_{k}}{c_{k}}=\frac{c_{L} \cdot 10^{k / n_{s}} \cdot\left(10^{1 / n_{s}}-1\right)}{c_{L} \cdot 10^{k / n_{s}}}=10^{1 / n_{s}}-1=\text { const } .
$$

The final expression for the joint probability distribution therefore becomes

$$
p\left(m_{1}, m_{2} \mid D\right) \propto \sum_{k=0}^{k_{H}}\left[\sum_{i=1}^{n}\left(U_{i}^{*}-c_{L} \cdot 10^{k / n_{s}} \cdot \Pi_{2, i}^{m_{1}} \cdot \Pi_{3, i}^{m_{2}}\right)^{2}\right]^{-n / 2}
$$

with $k_{H}=n_{s} \log _{10}\left(c_{H} / c_{L}\right)$. In this form, the joint probability distribution can be computed easily with very good resolution on a standard stand-alone computer.

\section{REFERENCES}

[1] Deardorff JW, Willis GE, Lilly DK. Laboratory investigation of non-steady penetrative convection. J Fluid Mech 1969; 35: 7-31.

[2] Willis GE, Deardorff JW. Laboratory model of the unstable planetary boundary layer. J Atmos Sci 1974; 31: 1297-307.

[3] Willis GE, Deardorff JW. Laboratory simulation of the convective planetary boundary layer. Atmos Technol 1975; 7: 80-6.

[4] Heidt FD. The growth of the mixed layer in a stratified fluid due to penetrative convection. Bound-Layer Meteor 1977; 12: 439-61.

[5] Deardorff JW, Willis GE, Stockton BH. Laboratory studies of the entrainment zone of a convectively mixed layer. J Fluid Mech 1980; 100: 41-64.

[6] Deardorff JW, Willis GE. Further results from a laboratory model of the convective planetary boundary layer. Bound-Layer Meteor $1985 ; 32: 205-36$

[7] Deardorff JW, Willis GE. Computer and laboratory modeling of the vertical diffusion of nonbuoyant particles in the mixed layer. Adv Geophys 1974; 18B: 187-200.

[8] Deardorff JW, Willis GE. Ground-level concentrations due to fumigation into an entraining mixed layer. Atmos Environ 1982; 16: 1159-70.

[9] Willis GE, Deardorff JW. On the use of Taylor's translation hypothesis for diffusion in the mixed layer. Q J R Meteor Soc 1976; 102: 817-22.

[10] Willis GE, Deardorff JW. Laboratory study of dispersion from an elevated source within a modelled convective planetary boundary layer. Atmos Environ 1978; 12: 1305-11.

[11] Willis GE, Deardorff JW. Laboratory study of dispersion from a source in the middle of the convectively mixed layer. Atmos Environ 1981; 15: 109-17.

[12] Willis GE, Deardorff JW. On plume rise within a convective boundary layer. Atmos Environ 1983; 17: 2435-47.
[13] Deardorff JW. Laboratory experiments on diffusion: The use of convective mixed-layer scaling. J Clim Appl Meteor 1985; 24(11): 1143-51

[14] Ohba R, Kakishima S, Ito S. Water tank experiment of gas diffusion from a stack in stably and unstably stratified layers under calm conditions. Atmos Environ 1991; 25: 2063-76.

[15] Hibberd MF, Sawford BL, Design criteria for water tank models of dispersion in the planetary convective boundary layer. BoundLayer Meteor 1994; 67: 97-118.

[16] Hibberd MF, Sawford BL. A saline laboratory model of the planetary convective boundary layer. Bound-Layer Meteor 1994; 67: 229-50.

[17] Luhar AK, Hibberd MF, Hurley PJ. Comparison of closure schemes used to specify the velocity PDF in Lagrangian stochastic dispersion models for convective conditions. Atmos Environ 1996; 30: $1407-18$

[18] Park OH, Seo SJ, Lee SH. Laboratory simulation of vertical plume dispersion within a convective boundary layer. Bound-Layer Meteor 2001; 99: 159-69.

[19] Snyder WH, Lawson RE Jr, Shipman MS, Lu J. Fluid modelling of atmospheric dispersion in the convective boundary layer. BoundLayer Meteor 2002; 102: 335-66.

[20] van Dop H, van As D, van Herwijnen A, Hibberd MF, Jonker H. Length scales of scalar diffusion in the convective boundary layer: laboratory observations. Bound-Layer Meteor 2005; 116: 1-35.

[21] Mitsumoto S, Ueda H, Ozoe H. A laboratory experiment on the dynamics of the land and sea breeze. J Atmos Sci 1983; 40: 122840 .

[22] Lu J, Arya SP, Snyder WH, Lawson RE Jr. A laboratory study of the urban heat island in a calm and stably stratified environment. Part I: Temperature field. J Appl Meteor 1997; 36: 1377-91.

[23] Lu J, Arya SP, Snyder WH, Lawson RE Jr. A laboratory study of the urban heat island in a calm and stably stratified environment. Part II: Velocity field. J Appl Meteor 1997; 36: 1392-402.

[24] Cenedese A, Monti P. Interaction between an inland urban heat island and a sea-breeze flow: A laboratory study. J Appl Meteor 2003; 42: 1569-83.

[25] Mitsumoto S. A laboratory experiment on the slope wind. J Meteor Soc Japan 1989; 67: 565-74

[26] Chen RR, Berman NS, Boyer DL, Fernando HJS. Physical model of diurnal heating in the vicinity of a two-dimensional ridge. J Atmos Sci 1996; 53: 62-85.

[27] Hunt JCR, Fernando HJS, Princevac M. Unsteady thermally driven flows on gentle slopes. J Atmos Sci 2003; 60: 2169-82.

[28] Deardorff JW, Willis GE. Turbulence within a baroclinic laboratory mixed layer above a sloping surface. J Atmos Sci 1987; 44: 772-78.

[29] Reuten C, Steyn DG, Allen SE. Water-Tank studies of atmospheric boundary layer structure and air-pollution transport in upslope flow systems. J Geophys Res 2007; 112: D11114.

[30] Reuten C, Steyn DG, Strawbridge KB, Bovis P. Observations of the relation between upslope flows and the convective boundary layer in steep terrain. Bound-Layer Meteor 2005; 116: 37-61.

[31] Garratt JR. The atmospheric boundary layer. 1st paperback ed. Cambridge Univ Press: Cambridge, UK 1994.

[32] Buckingham E. On physically similar systems; illustrations of the use of dimensional equations. Phys Rev Lett 1914; Second Series, IV: $345-376$

[33] Lilly DK. Models of cloud-topped mixed layers under a strong inversion. Q J R Meteor Soc 1968; 94: 292-309.

[34] Carson DJ. The development of a dry inversion-capped convectively unstable boundary layer. Q J R Meteor Soc 1973; 99: 450-67.

[35] Plate, EJ. Convective boundary layer: a historical introduction. In Buoyant Convection in Geophysical Flows. Plate EJ, et al., Eds. Kluwer Acad Pub 1998: 1-22.

[36] Schumann U. Large-eddy simulation of the upslope boundary layer. Q J R Meteor Soc 1990; 116: 637-70.

[37] Simpson JE. Gravity currents in the environment and the laboratory. $2^{\text {nd }}$ ed, Cambridge Univ Press: Cambridge, UK 1997.

[38] Britter RE, Linden PF. The motion of the front of a gravity current traveling down an incline. J Fluid Mech 1980; 99: 531-43.

[39] Reuten C. "Scaling and kinematics of daytime slope flow systems," The University of British Columbia, Vancouver, Canada, 2006. 
[40] Obasaju ED, Robins AG. Simulation of pollution dispersion using small scale physical models - An assessment of scaling options. Bound-Layer Meteor 1998; 52: 239-54.

[41] Schlichting H. Boundary-layer theory. $7^{\text {th }}$ ed, McGraw-Hill: Hamburg, Germany 1979.

[42] Hinze JO. Turbulence: An introduction to its mechanism and theory. $2^{\text {nd }}$ ed, McGraw-Hill: New York, USA 1975.

[43] Stull R. A convective transport theory for surface fluxes. J Atmos Sci 1994; 51: 3-22.

[44] Santoso E, Stull R. Convective transport theory: Further evaluation, and evidence of counter-difference surface heat fluxes. Boundary Layer Research Team Tech. Rep. BLRT-98-3, 1998. (Available from rstull@eos.ubc.ca)
[45] Greischar L, Stull R. Convective transport theory for surface fluxes tested over the western pacific warm pool. J Atmos Sci 1999; 56: 2201-11.

[46] Brutsaert W. Evaporation into the atmosphere. Kluwer: Dordrecht, The Netherlands 1982.

[47] Sivia DS. Data analysis. A Bayesian tutorial. Oxford University Press: Oxford, UK 1996.

[48] Jaynes ET. Probability theory. The logic of science. Cambridge Univ Press, Cambridge, USA 2003.

[49] Gelman A, Carlin JB, Stern HS, Rubin DB. Bayesian data analysis. 2nd ed, Chapman \& Hall/CRC: Boca Raton, USA 2004.

[50] Gregory P. Bayesian logical data analysis for the physical sciences. Cambridge Univ Press, Cambridge, UK 2005. 